\title{
Inadequate Filtration Process
}

National Cancer Institute

\section{Source}

National Cancer Institute. Inadequate Filtration Process. NCI Thesaurus. Code C62985.

Problem associated with the filter failing to remove items or substances which should have been removed. 